\title{
Good Lattice Rules with a Composite Number of Points Based on the Product Weighted Star Discrepancy
}

\author{
Vasile Sinescu and Stephen Joe \\ Department of Mathematics, University of Waikato, \\ Private Bag 3105, Hamilton, New Zealand \\ E-mails: vs27@math.waikato.ac.nz, stephenj@math.waikato.ac.nz
}

Summary. Rank-1 lattice rules based on a weighted star discrepancy with weights of a product form have been previously constructed under the assumption that the number of points is prime. Here, we extend these results to the non-prime case. We show that if the weights are summable, there exist lattice rules whose weighted star discrepancy is $O\left(n^{-1+\delta}\right)$, for any $\delta>0$, with the implied constant independent of the dimension and the number of lattice points, but dependent on $\delta$ and the weights. Then we show that the generating vector of such a rule can be constructed using a component-by-component $(\mathrm{CBC})$ technique. The cost of the $\mathrm{CBC}$ construction is analysed in the final part of the paper.

Key words: Rank-1 lattice rules, weighted star discrepancy, component-bycomponent construction.

\section{Introduction}

We consider rank-1 lattice rules for the approximation of integrals over the $d$-dimensional unit cube given by

$$
I_{d}(f)=\int_{[0,1]^{d}} f(\boldsymbol{x}) \mathrm{d} \boldsymbol{x} .
$$

These rank-1 lattice rules are quadrature rules of the form

$$
Q_{n, d}(f)=\frac{1}{n} \sum_{k=0}^{n-1} f\left(\left\{\frac{k \boldsymbol{z}}{n}\right\}\right)
$$

where $\boldsymbol{z} \in \mathbb{Z}^{d}$ is the generating vector having all the components conveniently assumed to be relatively prime with $n$, while the braces around a vector indicate that we take the fractional part of each component of the vector. 
In this paper we are looking to extend the recent results in [Joe06] by constructing rank-1 lattice rules with a composite number of points. Hence, the same assumptions as in [Joe06] will be used here with the main difference that $n$ is assumed to be just a positive integer. The vast majority of earlier research papers have assumed that $n$ was a prime number; an assumption which simplifies the analysis of the problem.

However there are some known results in the non-prime case. For instance, it has been proven in [Dis90], [Nie78], or [Nie92, Chapter 5] that good lattice rules with a non-prime number of points do exist. Several measures of goodness were used in those works, but under the assumptions that variables are equally important. Here, we assume that variables are arranged in the decreasing order of their importance and we employ a weighted star discrepancy as a criterion of goodness. An unweighted star discrepancy (corresponding to an $L_{\infty}$ maximum error) has been previously used in [Joe04] and in more general works such as [Nie92] or [SJ94], while the weighted star discrepancy has been used in [HN03], [Joe06], and [SJ07].

A constructive approach in the non-prime case has been proposed in [KJ02], where the integrands were assumed to belong to certain reproducing kernel Hilbert spaces such as weighted Korobov spaces of periodic functions or weighted Sobolev spaces with square-integrable mixed first derivatives. Here we require the integrands to have the weaker requirement of integrable mixed first derivatives. Let us remark that in [Kuo03] it was proven that in the reproducing kernel Hilbert spaces of [KJ02], the component-by-component construction (used also here) achieves the optimal rate of convergence. In [Dic04], the results in [Kuo03] were further improved and then extended to the non-prime case.

Let us also mention that lattice rules with a composite number of points have become more interesting since the introduction of extensible lattice rules in [HH97]. Later, in [HN03], it was shown that extensible lattice rules in number of points with a low weighted star discrepancy do exist, but the proof was non-constructive. More recently, in [DPW07], a possible way of constructing extensible lattice rules was proposed. Therein, it was assumed that $n$ is of the form $p^{m}$ with $p \geq 2$ an arbitrary prime. For such a case, it has been shown that lattice rules extensible in number of points based on the weighted star discrepancy can be constructed, but the results were not generalised to arbitrary integers as we propose here.

\section{Weighted Star Discrepancy}

As mentioned in the previous section, throughout this paper we make similar assumptions as in [Joe06] and we start by recalling some of those results and assumptions.

In order to introduce the general weighted star discrepancy, let us consider first the point set $P_{n}(\boldsymbol{z}):=\{\{k \boldsymbol{z} / n\}, 0 \leq k \leq n-1\}$. Then the local 
discrepancy of the point set $P_{n}(\boldsymbol{z})$ at $\boldsymbol{x} \in[0,1]^{d}$ is defined by

$$
\operatorname{discr}\left(\boldsymbol{x}, P_{n}\right):=\frac{A\left([\mathbf{0}, \boldsymbol{x}), P_{n}\right)}{n}-\prod_{j=1}^{d} x_{j}
$$

Here $A\left([\mathbf{0}, \boldsymbol{x}), P_{n}\right)$ represents the counting function, namely the number of points in $P_{n}(\boldsymbol{z})$ which lie in $[\mathbf{0}, \boldsymbol{x})$ with $\boldsymbol{x}=\left(x_{1}, x_{2}, \ldots, x_{d}\right)$.

Let now $\mathfrak{u}$ be an arbitrary non-empty subset of $\mathcal{D}:=\{1,2, \ldots, d-1, d\}$ and denote its cardinality by $|\mathfrak{u}|$. For the vector $\boldsymbol{x} \in[0,1]^{d}$, let $\boldsymbol{x}_{\mathfrak{u}}$ denote the vector from $[0,1]^{|\mathfrak{u}|}$ containing the components of $\boldsymbol{x}$ whose indices belong to $\mathfrak{u}$. By $\left(\boldsymbol{x}_{\mathfrak{u}}, \mathbf{1}\right)$ we mean the vector from $[0,1]^{d}$ whose $j$-th component is $x_{j}$ if $j \in \mathfrak{u}$ and 1 if $j \notin \mathfrak{u}$. From Zaremba's identity (see for instance [SW98] or [Zar68]), we obtain

$$
Q_{n, d}(f)-I_{d}(f)=\sum_{\mathfrak{u} \subseteq \mathcal{D}}(-1)^{|\mathfrak{u}|} \int_{[0,1]^{|\mathfrak{u}|}} \operatorname{discr}\left(\left(\boldsymbol{x}_{\mathfrak{u}}, \mathbf{1}\right), P_{n}\right) \frac{\partial^{|\mathfrak{u}|} f\left(\left(\boldsymbol{x}_{\mathfrak{u}}, \mathbf{1}\right)\right)}{\partial \boldsymbol{x}_{\mathfrak{u}}} \mathrm{d} \boldsymbol{x}_{\mathfrak{u}} .
$$

Now let us introduce a sequence of positive weights $\left\{\gamma_{j}\right\}_{j=1}^{\infty}$, which describe the decreasing importance of the successive coordinates $x_{j}$ and consider $\gamma_{\mathfrak{u}}$ as the weight associated with the set $\mathfrak{u}$. In this paper, we assume that the weights $\left\{\gamma_{\mathfrak{u}}\right\}$ are "product", that is

$$
\gamma_{\mathfrak{u}}=\prod_{j \in \mathfrak{u}} \gamma_{j},
$$

for any subset $\mathfrak{u} \subseteq \mathcal{D}$. Such assumptions on the weights have been made in [HN03], [Joe06], [SW98] and in other research papers. Using (1) we see that we can write

$$
\begin{aligned}
& Q_{n, d}(f)-I_{d}(f) \\
= & \sum_{\mathfrak{u} \subseteq \mathcal{D}}(-1)^{|\mathfrak{u}|} \gamma_{\mathfrak{u}} \int_{[0,1]^{|\mathfrak{u}|}} \operatorname{discr}\left(\left(\boldsymbol{x}_{\mathfrak{u}}, \mathbf{1}\right), P_{n}\right) \boldsymbol{\gamma}_{\mathfrak{u}}^{-1} \frac{\partial^{|\mathfrak{u}|} f\left(\left(\boldsymbol{x}_{\mathfrak{u}}, \mathbf{1}\right)\right)}{\partial \boldsymbol{x}_{\mathfrak{u}}} \mathrm{d} \boldsymbol{x}_{\mathfrak{u}} .
\end{aligned}
$$

Applying Hölder's inequality for integrals and sums, we obtain

$$
\begin{aligned}
\left|Q_{n, d}(f)-I_{d}(f)\right| & \leq\left(\sum_{\mathfrak{u} \subseteq \mathcal{D}} \sup _{\boldsymbol{x}_{\mathfrak{u}} \in[0,1]^{|\mathfrak{u}|}} \gamma_{\mathfrak{u}}\left|\operatorname{discr}\left(\left(\boldsymbol{x}_{\mathfrak{u}}, \mathbf{1}\right), P_{n}\right)\right|\right) \\
& \times\left(\max _{\mathfrak{u} \subseteq \mathcal{D}} \gamma_{\mathfrak{u}}^{-1} \int_{[0,1]^{|\mathfrak{u}|}}\left|\frac{\partial^{|\mathfrak{u}|}}{\partial \boldsymbol{x}_{\mathfrak{u}}} f\left(\left(\boldsymbol{x}_{\mathfrak{u}}, \mathbf{1}\right)\right)\right| \mathrm{d} \boldsymbol{x}_{\mathfrak{u}}\right) .
\end{aligned}
$$

Thus we can define a weighted star discrepancy $D_{n, \boldsymbol{\gamma}}^{*}(\boldsymbol{z})$ by

$$
D_{n, \boldsymbol{\gamma}}^{*}(\boldsymbol{z}):=\sum_{\mathfrak{u} \subseteq \mathcal{D}} \gamma_{\mathfrak{u}} \sup _{\boldsymbol{x}_{\mathfrak{u}} \in[0,1]^{|\mathfrak{u}|}}\left|\operatorname{discr}\left(\left(\boldsymbol{x}_{\mathfrak{u}}, \mathbf{1}\right), P_{n}\right)\right| .
$$


From [Nie92, Theorem 3.10 and Theorem 5.6], we obtain the following inequality:

$$
\sup _{\boldsymbol{x}_{\mathfrak{u}} \in[0,1]^{|\mathfrak{u}|}}\left|\operatorname{discr}\left(\left(\boldsymbol{x}_{\mathfrak{u}}, \mathbf{1}\right), P_{n}\right)\right| \leq 1-(1-1 / n)^{|\mathfrak{u}|}+\frac{R_{n}(\boldsymbol{z}, \mathfrak{u})}{2},
$$

where

$$
R_{n}(\boldsymbol{z}, \mathfrak{u})=\sum_{\substack{\boldsymbol{h} \cdot \boldsymbol{z}_{\mathfrak{u}} \equiv 0\left(\bmod \\ \boldsymbol{h} \in E_{n,|\mathfrak{u}|}^{*}\right.}} \prod_{j \in \mathfrak{u}} \frac{1}{\max \left(1,\left|h_{j}\right|\right)} .
$$

Here $\boldsymbol{z}_{\mathfrak{u}}$ denotes the vector consisting of the components of $\boldsymbol{z}$ whose indices belong to $\mathfrak{u}$, while

$$
E_{n, m}^{*}=\left\{\boldsymbol{h} \in \mathbb{Z}^{m}, \boldsymbol{h} \neq \mathbf{0}:-n / 2<h_{j} \leq n / 2,1 \leq j \leq m\right\} .
$$

This result, together with (2) shows that the general weighted star discrepancy satisfies the inequality

$$
D_{n, \boldsymbol{\gamma}}^{*}(\boldsymbol{z}) \leq \sum_{\mathfrak{u} \subseteq \mathcal{D}} \gamma_{\mathfrak{u}}\left(1-(1-1 / n)^{|\mathfrak{u}|}+\frac{R_{n}(\boldsymbol{z}, \mathfrak{u})}{2}\right) .
$$

For calculation purposes, the theory of lattice rules, (for example, see [Nie92] or [SJ94]) shows that we may write $R_{n}(\boldsymbol{z}, \mathfrak{u})$ as

$$
R_{n}(\boldsymbol{z}, \mathfrak{u})=\frac{1}{n} \sum_{k=0}^{n-1} \prod_{j \in \mathfrak{u}}\left(1+\sum_{-n / 2<h \leq n / 2}^{\prime} \frac{e^{2 \pi \mathrm{i} h k z_{j} / n}}{|h|}\right)-1,
$$

where the ' in the sum indicates we omit the $h=0$ term. It is easy to see that $R_{n}(\boldsymbol{z}, \mathfrak{u})$ represents the quadrature error produced by applying the lattice rule to the integrand

$$
\prod_{j \in \mathfrak{u}}\left(1+\sum_{-n / 2<h \leq n / 2}^{\prime} \frac{e^{2 \pi \mathrm{i} h x_{j}}}{|h|}\right) .
$$

It is also easy to check that if $|\mathfrak{u}|=1$, then the corresponding error $R_{n}(\boldsymbol{z}, \mathfrak{u})=$ 0 for each subset of $\mathcal{D}$ with only one element.

\section{Bounds on the Weighted Star Discrepancy}

To obtain bounds on $D_{n, \gamma}^{*}(\boldsymbol{z})$, we see from (3) that we need to bound the quantity

$$
\sum_{\mathfrak{u} \subseteq \mathcal{D}} \gamma_{\mathfrak{u}}\left(1-(1-1 / n)^{|\mathfrak{u}|}\right)
$$

and the quantity 


$$
e_{n, d}^{2}(\boldsymbol{z}):=\sum_{\mathfrak{u} \subseteq \mathcal{D}} \gamma_{\mathfrak{u}} R_{n}(\boldsymbol{z}, \mathfrak{u}) .
$$

Under the assumption that the weights are summable, that is $\sum_{j=1}^{\infty} \gamma_{j}<\infty$, it follows from [Joe06, Lemma 1] that

$$
\sum_{\mathfrak{u} \subseteq \mathcal{D}} \gamma_{\mathfrak{u}}\left(1-(1-1 / n)^{|\mathfrak{u}|}\right)=O\left(n^{-1}\right),
$$

with the implied constant depending on the weights, but independent of $d$ and $n$.

We now consider $e_{n, d}^{2}(\boldsymbol{z})$ in more detail and by expanding the quadrature error defined by (4) as in [Joe06], we obtain

$$
e_{n, d}^{2}(\boldsymbol{z})=\frac{1}{n} \sum_{k=0}^{n-1} \prod_{j=1}^{d}\left(1+\gamma_{j}+\gamma_{j} C_{k}\left(z_{j}\right)\right)-\prod_{j=1}^{d}\left(1+\gamma_{j}\right),
$$

where

$$
C_{k}(z)=\sum_{-n / 2<h \leq n / 2}^{\prime} \frac{e^{2 \pi \mathrm{i} h k z / n}}{|h|} .
$$

By setting $\beta_{j}=1+\gamma_{j}$, we obtain

$$
e_{n, d}^{2}(\boldsymbol{z})=\frac{1}{n} \sum_{k=0}^{n-1} \prod_{j=1}^{d}\left(\beta_{j}+\gamma_{j} C_{k}\left(z_{j}\right)\right)-\prod_{j=1}^{d} \beta_{j} .
$$

We can obtain a bound on $e_{n, d}^{2}(\boldsymbol{z})$ by obtaining a bound on a certain mean value of $e_{n, d}^{2}(\boldsymbol{z})$. The mean $M_{n, d, \gamma}$ is defined by

$$
M_{n, d, \boldsymbol{\gamma}}:=\frac{1}{\varphi(n)^{d}} \sum_{\boldsymbol{z} \in \mathcal{Z}_{n}^{d}} e_{n, d}^{2}(\boldsymbol{z}),
$$

where $\varphi$ is Euler's totient function and

$$
\mathcal{Z}_{n}=\{z: z \in\{1,2, \ldots, n-1\},(z, n)=1\}
$$

has cardinality $\varphi(n)$. Here $(z, n)=\operatorname{gcd}(z, n)$. A bound on the mean $M_{n, d, \gamma}$ is given next.

Theorem 1. Let $n \geq 2$ be an integer and let

$$
S_{n}=\sum_{-n / 2<h \leq n / 2}^{\prime} \frac{1}{|h|} \text {. }
$$

If the weights $\left\{\gamma_{j}\right\}_{j=1}^{\infty}$ are summable, then 


$$
M_{n, d, \boldsymbol{\gamma}} \leq \frac{1}{n} \prod_{j=1}^{d}\left(\beta_{j}+\gamma_{j} S_{n}\right)+O\left(\frac{\ln \ln (n+1)}{n}\right),
$$

where the implied constant depends on the weights, but is independent of the dimension.

Proof. We have

$$
\begin{aligned}
M_{n, d, \gamma} & =\frac{1}{\varphi(n)^{d}} \sum_{z \in \mathcal{Z}_{n}^{d}}\left(\frac{1}{n} \sum_{k=0}^{n-1} \prod_{j=1}^{d}\left(\beta_{j}+\gamma_{j} C_{k}\left(z_{j}\right)\right)-\prod_{j=1}^{d} \beta_{j}\right) \\
& =\frac{1}{n} \sum_{k=0}^{n-1} \prod_{j=1}^{d}\left(\frac{1}{\varphi(n)} \sum_{z_{j} \in \mathcal{Z}_{n}}\left(\beta_{j}+\gamma_{j} C_{k}\left(z_{j}\right)\right)\right)-\prod_{j=1}^{d} \beta_{j} \\
& =\frac{1}{n} \prod_{j=1}^{d}\left(\beta_{j}+\gamma_{j} S_{n}\right)+\frac{1}{n} \sum_{k=1}^{n-1} \prod_{j=1}^{d}\left(\beta_{j}+\frac{\gamma_{j}}{\varphi(n)} \sum_{z_{j} \in \mathcal{Z}_{n}} C_{k}\left(z_{j}\right)\right)-\prod_{j=1}^{d} \beta_{j},
\end{aligned}
$$

where in the last step the $k=0$ term has been separated out and we have used the fact that $C_{0}(z)=S_{n}$. If we denote

$$
T_{n}(k)=\sum_{z \in \mathcal{Z}_{n}} C_{k}(z)=\sum_{z \in \mathcal{Z}_{n}} \sum_{-n / 2<h \leq n / 2}^{\prime} \frac{e^{2 \pi \mathrm{i} h k z / n}}{|h|},
$$

then we see that the mean can be written as

$$
M_{n, d, \boldsymbol{\gamma}}=\frac{1}{n} \prod_{j=1}^{d}\left(\beta_{j}+\gamma_{j} S_{n}\right)+L_{n, d, \boldsymbol{\gamma}}-\prod_{j=1}^{d} \beta_{j},
$$

where

$$
L_{n, d, \boldsymbol{\gamma}}=\frac{1}{n} \sum_{k=1}^{n-1} \prod_{j=1}^{d}\left(\beta_{j}+\frac{\gamma_{j}}{\varphi(n)} T_{n}(k)\right) .
$$

The rest of this proof follows many of the arguments used in the proof of [Nie92, Theorem 5.10] (see also [Dis90]). Firstly, it may be shown that

$$
T_{n}(k)=\sum_{a \mid n} \mu(a)\left(\frac{n}{a}, k\right) S_{a\left(\frac{n}{a}, k\right)}=\sum_{a \mid n} \mu\left(\frac{n}{a}\right)(a, k) S_{\frac{n(a, k)}{a}},
$$

where $\mu$ denotes the well-known Möbius function from number theory. If $n$ is prime, then we obtain $T_{n}(k)=-S_{n}$ for any $1 \leq k \leq n-1$, which leads to the results obtained in [Joe06]. From [Nie78, Lemmas 1 and 2], we have

$$
S_{m}=2 \ln m+2 \omega-\ln 4+\varepsilon(m),
$$

where $\omega$ is the Euler-Mascheroni constant given by 


$$
\omega=\lim _{\ell \rightarrow \infty}\left(\sum_{k=1}^{\ell} \frac{1}{k}-\ln \ell\right)
$$

while

$$
|\varepsilon(m)|<4 m^{-2}
$$

Using (10), we now obtain

$$
T_{n}(k)=(2 \ln n+2 \omega-\ln 4) B_{n}(k)-2 H_{n}(k)+V_{n}(k),
$$

where

$$
\begin{gathered}
B_{n}(k)=\sum_{a \mid n} \mu\left(\frac{n}{a}\right)(a, k), \\
H_{n}(k)=\sum_{a \mid n} \mu\left(\frac{n}{a}\right)(a, k) \ln \frac{a}{(a, k)},
\end{gathered}
$$

and

$$
V_{n}(k)=\sum_{a \mid n} \mu\left(\frac{n}{a}\right)(a, k) \varepsilon\left(\frac{n(a, k)}{a}\right) .
$$

From the proof of [Nie92, Theorem 5.10], we have $B_{n}(k)=0$ for any $1 \leq k \leq$ $n-1$. Using this result in (13), we get

$$
T_{n}(k)=-2 H_{n}(k)+V_{n}(k) .
$$

By combining (9) with (15), we obtain

$$
L_{n, d, \boldsymbol{\gamma}}=\frac{1}{n} \sum_{k=1}^{n-1} \prod_{j=1}^{d}\left(\beta_{j}+\gamma_{j}\left(-2 J_{n}(k)+\frac{V_{n}(k)}{\varphi(n)}\right)\right),
$$

where

$$
J_{n}(k)=\frac{H_{n}(k)}{\varphi(n)} .
$$

The proof of Theorem 5.10 in [Nie92] yields $V_{n}(k)=O(1)$ with an absolute implied constant. Hence we have $V_{n}(k) / \varphi(n)=O(1 / \varphi(n))$. This result together with (16) and $\beta_{j}=1+\gamma_{j}$ yields

$$
L_{n, d, \boldsymbol{\gamma}}=\frac{1}{n} \sum_{k=1}^{n-1} \prod_{j=1}^{d}\left(1+\gamma_{j}\left(1-2 J_{n}(k)\right)+\gamma_{j} O\left(\frac{1}{\varphi(n)}\right)\right) .
$$

Let us denote by $p$ a prime number and by $e_{p}(n)$ the largest exponent such that $p^{e_{p}(n)}$ divides $n$. Then, from the proof of [Nie92, Theorem 5.10], we obtain $H_{n}(k)= \begin{cases}p^{e_{p}(k)} \varphi\left(n / p^{e_{p}(n)}\right) \ln p, & \text { if } p \text { is the unique prime with } e_{p}(n)>e_{p}(k), \\ 0, & \text { otherwise }\end{cases}$ 
If such a $p$ exists, then by the definition of $e_{p}(n)$, we have $n / p^{e_{p}(n)}$ relatively prime with $p^{e_{p}(n)}$ and hence $\varphi\left(n / p^{e_{p}(n)}\right) \varphi\left(p^{e_{p}(n)}\right)=\varphi(n)$. We then obtain

$$
J_{n}(k)=\frac{p^{e_{p}(k)} \varphi\left(n / p^{e_{p}(n)}\right) \ln p}{\varphi(n)}=\frac{p^{e_{p}(k)} \ln p}{\varphi\left(p^{e_{p}}(n)\right.}=\frac{\ln p}{p^{\alpha_{k}}(p-1)},
$$

where we put $\alpha_{k}=e_{p}(n)-e_{p}(k)-1$, for $1 \leq k \leq n-1$. For each $1 \leq k \leq n-1$, it is not difficult to check from (18) that $-1<1-2 \ln (p) /\left(p^{\alpha_{k}}(p-1)\right)<1$ for any prime $p \geq 2$ and for any $\alpha_{k} \geq 0$. Hence, $1+\gamma_{j}\left(1-2 J_{n}(k)\right) \leq 1+\gamma_{j}=\beta_{j}$ for any $1 \leq j \leq d$. The product in (17) can then be bounded by

$$
\begin{aligned}
& \prod_{j=1}^{d}\left(1+\gamma_{j}\left(1-2 J_{n}(k)\right)+\gamma_{j} O\left(\frac{1}{\varphi(n)}\right)\right) \\
\leq & \prod_{j=1}^{d}\left(\beta_{j}+\gamma_{j} O\left(\frac{1}{\varphi(n)}\right)\right) \\
= & \prod_{j=1}^{d} \beta_{j}+\sum_{\substack{\mathfrak{u} \subseteq \mathcal{D} \\
|\mathfrak{u}| \geq 1}}\left(O\left(\frac{1}{\varphi(n)}\right)\right)^{|\mathfrak{u}|} \prod_{j \in \mathfrak{u}} \gamma_{j} \prod_{j \notin \mathfrak{u}} \beta_{j} \\
= & \prod_{j=1}^{d} \beta_{j}+O\left(\frac{1}{\varphi(n)}\right),
\end{aligned}
$$

where the implied constant depends on the quantity

$$
\sum_{\substack{\mathfrak{u} \subseteq \mathcal{D} \\|\mathfrak{u}| \geq 1}} \prod_{j \in \mathfrak{u}} \gamma_{j} \prod_{j \notin \mathfrak{u}} \beta_{j} \leq \prod_{j=1}^{d}\left(\beta_{j}+\gamma_{j}\right)
$$

Next, let us consider

$$
\prod_{j=1}^{d}\left(\beta_{j}+\gamma_{j}\right)=\exp \left(\sum_{j=1}^{d} \ln \left(\beta_{j}+\gamma_{j}\right)\right) \leq \exp \left(2 \sum_{j=1}^{d} \gamma_{j}\right),
$$

where we used that $\beta_{j}=1+\gamma_{j}$ and $\ln (1+x) \leq x$ for any $x>-1$. Recalling that the weights were assumed to be summable, by denoting $\Gamma:=\sum_{j=1}^{\infty} \gamma_{j}$, it follows that

$$
\prod_{j=1}^{d}\left(\beta_{j}+\gamma_{j}\right) \leq e^{2 \Gamma}
$$

which shows that the implied constant of (19) is independent of the dimension, but dependent on the weights. From (17), (19) and using that $1 / \varphi(n)=$ $O\left(n^{-1} \ln \ln (n+1)\right)$, we now obtain 


$$
L_{n, d, \gamma} \leq \frac{n-1}{n} \prod_{j=1}^{d} \beta_{j}+O\left(\frac{\ln \ln (n+1)}{n}\right) .
$$

By combining the last inequality with (8), we obtain

$$
M_{n, d, \gamma} \leq \frac{1}{n} \prod_{j=1}^{d}\left(\beta_{j}+\gamma_{j} S_{n}\right)+O\left(\frac{\ln \ln (n+1)}{n}\right) .
$$

Corollary 1. Let $n \geq 2$ be an integer. If the weights $\left\{\gamma_{j}\right\}_{j=1}^{\infty}$ are summable, then there exists a vector $\boldsymbol{z} \in \mathcal{Z}_{n}^{d}$ such that

$$
e_{n, d}^{2}(\boldsymbol{z}) \leq \frac{1}{n} \prod_{j=1}^{d}\left(\beta_{j}+\gamma_{j} S_{n}\right)+O\left(\frac{\ln \ln (n+1)}{n}\right),
$$

where the implied constant depends on the weights, but is independent of the dimension.

Proof. Clearly, there must be a vector $\boldsymbol{z} \in \mathcal{Z}_{n}^{d}$ such that $e_{n, d}^{2}(\boldsymbol{z}) \leq M_{n, d, \boldsymbol{\gamma}}$ and the result then follows from Theorem 1 .

It is known from [Nie78] or [Nie92] that in an unweighted setting there exist $d$-dimensional lattice rules having $O\left(n^{-1}(\ln n)^{d}\right)$ star discrepancy with the implied constant depending only on $d$. Such a bound is widely believed to be the best possible (see [Lar87] or [Nie92] for details). In our situation, from (3), (5) and Corollary 1, together with the observation that $S_{n} \leq 2 \ln n$ for any $n \geq 2$ (this follows from (11) for $n \geq 3$ and a direct calculation for $n=2$ ), it will follow that there exists a vector $\boldsymbol{z} \in \mathcal{Z}_{n}^{d}$ such that

$$
D_{n, \boldsymbol{\gamma}}^{*}(\boldsymbol{z})=O\left(n^{-1}(\ln n)^{d}\right),
$$

but with the implied constant independent of $d$. A bound that does not involve $\ln n$ is possible by making use of [HN03, Lemma 3]. This result leads to the conclusion that if the weights are summable, then there exists a generating vector $\boldsymbol{z}$ such that the weighted star discrepancy achieves the error bound

$$
D_{n, \boldsymbol{\gamma}}^{*}(\boldsymbol{z})=O\left(n^{-1+\delta}\right),
$$

for any $\delta>0$, where the implied constant depends on $\delta$ and the weights but is independent of $n$ and $d$.

Let us also remark that corresponding results for a weighted $L_{p}$ star discrepancy can be deduced, since such a discrepancy is bounded by the discrepancy introduced in (2). Further details can be found in [Joe06]. 


\section{A Component-by-Component Construction}

Before presenting the main result regarding the $\mathrm{CBC}$ construction, we need the following:

Lemma 1. There exists a positive constant $c$ independent of $n$ such that

$$
\sum_{k=1}^{n-1} \frac{\left|T_{n}(k)\right|}{\varphi(n)} \leq c \ln n
$$

where $T_{n}(k)$ has been defined by $(7)$.

Proof. Since $J_{n}(k)=H_{n}(k) / \varphi(n) \geq 0$, then from (15), we obtain:

$$
\sum_{k=1}^{n-1} \frac{\left|T_{n}(k)\right|}{\varphi(n)} \leq \sum_{k=1}^{n-1}\left(2 J_{n}(k)+\frac{\left|V_{n}(k)\right|}{\varphi(n)}\right) .
$$

From the proof of [Nie92, Theorem 5.10], we obtain:

$$
\sum_{k=1}^{n-1} J_{n}(k)=\ln n
$$

In order to analyse the second quantity of (20), we see from (14) that

$$
\left|V_{n}(k)\right| \leq \sum_{a \mid n}\left|\mu\left(\frac{n}{a}\right)\right|(a, k)\left|\varepsilon\left(\frac{n(a, k)}{a}\right)\right| .
$$

By using (12), we next obtain:

$$
\left|V_{n}(k)\right| \leq 4 \sum_{a \mid n}\left|\mu\left(\frac{n}{a}\right)\right|\left(\frac{a}{n}\right)^{2}=4 \sum_{a \mid n} \frac{1}{a^{2}} \leq \frac{2 \pi^{2}}{3} .
$$

Recalling that $1 / \varphi(n)=O(\ln \ln (n+1) / n)$ with an absolute implied constant, we now deduce that there exists a constant $c_{1}>0$ independent of $n$ such that

$$
\sum_{k=1}^{n-1} \frac{\left|V_{n}(k)\right|}{\varphi(n)} \leq(n-1) \frac{2 \pi^{2} c_{1}}{3} \frac{\ln \ln (n+1)}{n} \leq \frac{2 \pi^{2} c_{1} \ln n}{3}
$$

From this inequality combined with (20) and (21), we obtain:

$$
\sum_{k=1}^{n-1} \frac{\left|T_{n}(k)\right|}{\varphi(n)} \leq\left(2+\frac{2 \pi^{2} c_{1}}{3}\right) \ln n
$$

which leads to the desired result by taking $c=2+2 \pi^{2} c_{1} / 3$. 
In order to construct the generating vector, we use a component-bycomponent (CBC) technique, which is essentially a "greedy"-type algorithm, based on finding each component one at a time. This technique has been successfully used in several research papers, for instance [Joe04], [Joe06] or [KJ02]. Here, we are looking to prove that the CBC algorithm produces a generating vector whose corresponding weighted star discrepancy has the same order of magnitude as the bound given in Corollary 1. The CBC algorithm is presented below:

\section{Component-by-component (CBC) algorithm}

Assume that $n \geq 2$ is an integer, $d$ is the dimension and all the weights are known. Then the generating vector $\boldsymbol{z}=\left(z_{1}, z_{2}, \ldots, z_{d}\right)$ can be constructed as follows:

1. Set the value for the first component of the vector, say $z_{1}:=1$.

2. For $m=2,3, \ldots, d$, find $z_{m} \in \mathcal{Z}_{n}$ such that $e_{n, m}^{2}\left(z_{1}, z_{2}, \ldots, z_{m}\right)$ is minimised.

In the above, we have

$$
e_{n, m}^{2}\left(z_{1}, z_{2}, \ldots, z_{m}\right)=\frac{1}{n} \sum_{k=0}^{n-1} \prod_{j=1}^{m}\left(\beta_{j}+\gamma_{j} C_{k}\left(z_{j}\right)\right)-\prod_{j=1}^{m} \beta_{j} .
$$

The following theorem and corollary will justify the use of the CBC algorithm.

Theorem 2. Let $n \geq 2$ be an integer and suppose that the weights $\left\{\gamma_{j}\right\}_{j=1}^{\infty}$ are summable. If there exists a $\boldsymbol{z} \in \mathcal{Z}_{n}^{d}$ such that

$$
e_{n, d}^{2}(\boldsymbol{z}) \leq \frac{1}{n} \prod_{j=1}^{d}\left(\beta_{j}+\alpha \gamma_{j} \ln n\right)
$$

where $\alpha=2+c$ with $c$ defined by Lemma 1 , then there exists $z_{d+1} \in \mathcal{Z}_{n}$ such that

$$
e_{n, d+1}^{2}\left(\boldsymbol{z}, z_{d+1}\right) \leq \frac{1}{n} \prod_{j=1}^{d+1}\left(\beta_{j}+\alpha \gamma_{j} \ln n\right) .
$$

Such a $z_{d+1}$ can be found by minimising $e_{n, d+1}^{2}\left(\boldsymbol{z}, z_{d+1}\right)$ over the set $\mathcal{Z}_{n}$.

Proof. For any $z_{d+1} \in \mathcal{Z}_{n}$, we see from (6) that

$$
\begin{aligned}
e_{n, d+1}^{2}\left(\boldsymbol{z}, z_{d+1}\right) & =\frac{1}{n} \sum_{k=0}^{n-1} \prod_{j=1}^{d}\left(\beta_{j}+\gamma_{j} C_{k}\left(z_{j}\right)\right)\left(\beta_{d+1}+\gamma_{d+1} C_{k}\left(z_{d+1}\right)\right)-\beta_{d+1} \prod_{j=1}^{d} \beta_{j} \\
& =\beta_{d+1} e_{n, d}^{2}(\boldsymbol{z})+\frac{\gamma_{d+1}}{n} \sum_{k=0}^{n-1} \prod_{j=1}^{d}\left(\beta_{j}+\gamma_{j} C_{k}\left(z_{j}\right)\right) C_{k}\left(z_{d+1}\right) \\
& =\beta_{d+1} e_{n, d}^{2}(\boldsymbol{z})+\frac{\gamma_{d+1} S_{n}}{n} \prod_{j=1}^{d}\left(\beta_{j}+\gamma_{j} S_{n}\right)
\end{aligned}
$$




$$
+\frac{\gamma_{d+1}}{n} \sum_{k=1}^{n-1} \prod_{j=1}^{d}\left(\beta_{j}+\gamma_{j} C_{k}\left(z_{j}\right)\right) C_{k}\left(z_{d+1}\right),
$$

where in the last step the $k=0$ term has been separated out. Next we average $e_{n, d+1}^{2}\left(\boldsymbol{z}, z_{d+1}\right)$ over all the possible values of $z_{d+1}$ to form

$$
\begin{aligned}
\operatorname{Avg}\left(e_{n, d+1}^{2}\left(\boldsymbol{z}, z_{d+1}\right)\right)= & \frac{1}{\varphi(n)} \sum_{z_{d+1} \in \mathcal{Z}_{n}} e_{n, d+1}^{2}\left(\boldsymbol{z}, z_{d+1}\right) \\
= & \beta_{d+1} e_{n, d}^{2}(\boldsymbol{z})+\frac{\gamma_{d+1} S_{n}}{n} \prod_{j=1}^{d}\left(\beta_{j}+\gamma_{j} S_{n}\right) \\
& +\frac{\gamma_{d+1}}{n \varphi(n)} \sum_{z_{d+1} \in \mathcal{Z}_{n}} \sum_{k=1}^{n-1} \prod_{j=1}^{d}\left(\beta_{j}+\gamma_{j} C_{k}\left(z_{j}\right)\right) C_{k}\left(z_{d+1}\right) \\
= & \beta_{d+1} e_{n, d}^{2}(\boldsymbol{z})+\frac{\gamma_{d+1} S_{n}}{n} \prod_{j=1}^{d}\left(\beta_{j}+\gamma_{j} S_{n}\right) \\
& +\frac{\gamma_{d+1}}{n} \sum_{k=1}^{n-1}\left(\frac{1}{\varphi(n)} \sum_{z_{d+1} \in \mathcal{Z}_{n}} C_{k}\left(z_{d+1}\right)\right) \prod_{j=1}^{d}\left(\beta_{j}+\gamma_{j} C_{k}\left(z_{j}\right)\right) \\
\leq & \beta_{d+1} e_{n, d}^{2}(\boldsymbol{z})+\frac{\gamma_{d+1} S_{n}}{n} \prod_{j=1}^{d}\left(\beta_{j}+\gamma_{j} S_{n}\right) \\
& +\frac{\gamma_{d+1}}{n} \sum_{k=1}^{n-1} \frac{\left|T_{n}(k)\right|}{\varphi(n)} \prod_{j=1}^{d}\left(\beta_{j}+\gamma_{j} S_{n}\right) .
\end{aligned}
$$

Using Lemma 1 and $S_{n} \leq 2 \ln n$, we next obtain

$$
\begin{aligned}
\operatorname{Avg}\left(e_{n, d+1}^{2}\left(\boldsymbol{z}, z_{d+1}\right)\right) \leq & \beta_{d+1} e_{n, d}^{2}(\boldsymbol{z})+\frac{\gamma_{d+1} S_{n}}{n} \prod_{j=1}^{d}\left(\beta_{j}+\gamma_{j} S_{n}\right) \\
& +\frac{c \gamma_{d+1} \ln n}{n} \prod_{j=1}^{d}\left(\beta_{j}+\gamma_{j} S_{n}\right) \\
\leq & \beta_{d+1} e_{n, d}^{2}(\boldsymbol{z})+\frac{(2+c) \gamma_{d+1} \ln n}{n} \prod_{j=1}^{d}\left(\beta_{j}+\gamma_{j} S_{n}\right) \\
\leq & \beta_{d+1} e_{n, d}^{2}(\boldsymbol{z})+\frac{\alpha \gamma_{d+1} \ln n}{n} \prod_{j=1}^{d}\left(\beta_{j}+\alpha \gamma_{j} \ln n\right) .
\end{aligned}
$$

By making use of the hypothesis, we finally obtain

$\operatorname{Avg}\left(e_{n, d+1}^{2}\left(z, z_{d+1}\right)\right) \leq \frac{\beta_{d+1}}{n} \prod_{j=1}^{d}\left(\beta_{j}+\alpha \gamma_{j} \ln n\right)+\frac{\alpha \gamma_{d+1} \ln n}{n} \prod_{j=1}^{d}\left(\beta_{j}+\alpha \gamma_{j} \ln n\right)$ 


$$
=\frac{1}{n} \prod_{j=1}^{d+1}\left(\beta_{j}+\alpha \gamma_{j} \ln n\right)
$$

It is obvious that the $z_{d+1} \in \mathcal{Z}_{n}$ chosen to minimise $e_{n, d+1}^{2}\left(\boldsymbol{z}, z_{d+1}\right)$ will satisfy

$$
e_{n, d+1}^{2}\left(\boldsymbol{z}, z_{d+1}\right) \leq \operatorname{Avg}\left(e_{n, d+1}^{2}\left(\boldsymbol{z}, z_{d+1}\right)\right) .
$$

This, together with the previous inequality completes the proof.

Corollary 2. Let $n \geq 2$ be an integer. If the weights $\left\{\gamma_{j}\right\}_{j=1}^{\infty}$ are summable, then for any $m=1,2, \ldots, d$, there exists a $\boldsymbol{z} \in \mathcal{Z}_{n}^{m}$ such that

$$
e_{n, m}^{2}\left(z_{1}, z_{2}, \ldots, z_{m}\right) \leq \frac{1}{n} \prod_{j=1}^{m}\left(\beta_{j}+\alpha \gamma_{j} S_{n}\right) .
$$

We can set $z_{1}=1$ and for every $2 \leq m \leq d, z_{m}$ can be chosen by minimising $e_{n, m}^{2}\left(z_{1}, z_{2}, \ldots, z_{m}\right)$ over the set $\mathcal{Z}_{n}$.

Proof. Recall from Section 2 that $R_{n}(\boldsymbol{z}, \mathfrak{u})=0$ for any subset $\mathfrak{u} \subseteq \mathcal{D}$ with $|\mathfrak{u}|=1$. Hence for $m=1$ it follows that $e_{n, 1}^{2}\left(z_{1}\right)=0$. The result then follows immediately from Theorem 2 .

In order to evaluate the complexity of the $\mathrm{CBC}$ construction, we observe first that each $e_{n, m}^{2}\left(z_{1}, z_{2}, \ldots, z_{m}\right)$ can be evaluated in $O\left(n^{2} m\right)$ operations. This cost can be reduced to $O(\mathrm{~nm})$ by using asymptotic techniques as presented in [JS92] (see also [Joe06, Appendix A]) and consequently, the total complexity of the algorithm will be $O\left(n^{2} d^{2}\right)$. This can be reduced to $O\left(n^{2} d\right)$ if we store the products during the construction at an extra expense of $O(n)$. However, this order of magnitude can be further reduced to $O(n d \log n)$ with an approach similar to the one used by Nuyens and Cools in [NC05]. Their approach is essentially based on a fast matrix-vector multiplication and consists of minimising a function of the form

$$
\frac{1}{n} \sum_{k=0}^{n-1} \prod_{j=1}^{d}\left(1+\gamma_{j} \omega\left(\left\{\frac{k z_{j}}{n}\right\}\right)\right)-1
$$

where $\omega$ is some function. In our situation we can take

$$
\omega(x)=\sum_{-\frac{n}{2}<h \leq \frac{n}{2}}^{\prime} \frac{e^{2 \pi \mathrm{i} h x}}{|h|}, x \in[0,1] .
$$

Thus, with some modifications, the techniques used in [NC05] will also work here. 


\section{References}

[Dic04] J. Dick. On the convergence rate of the component-by-component construction of good lattice rules. J. Complexity, 20: 493-522, 2004.

[Dis90] S. Disney. Error bounds for rank-1 lattice quadrature rules modulo composites. Monatsh. Math., 110: 89-100, 1990.

[DPW07] J. Dick, F. Pillichshammer, and B.J. Waterhouse. The construction of good extensible rank-1 lattices. Math. Comp., 2007. to appear.

[HH97] F.J. Hickernell and H.S. Hong. Computing multivariate normal probabilities using rank-1 lattice sequences. In G.H. Golub, S.H. Lui, F.T. Luk, and R.J. Plemmons, editors, Scientific Computing (Hong Kong, 1997), pages 209-215. Springer, Singapore, 1997.

[HN03] F.J. Hickernell and H. Niederreiter. The existence of good extensible rank1 lattices. J. Complexity, 19: 286-300, 2003.

[Joe04] S. Joe. Component by component construction of rank-1 lattice rules having $O\left(n^{-1}(\ln n)^{d}\right)$ star discrepancy. In H. Niederreiter, editor, Monte Carlo and Quasi-Monte Carlo Methods 2002, pages 293-298. Springer, 2004.

[Joe06] S. Joe. Construction of good rank-1 lattice rules based on the weighted star discrepancy. In H. Niederreiter and D. Talay, editors, Monte Carlo and Quasi-Monte Carlo Methods 2004, pages 181-196. Springer, 2006.

[JS92] S. Joe and I.H. Sloan. On computing the lattice rule criterion R. Math. Comp, 59: 557-568, 1992.

[KJ02] F.Y. Kuo and S. Joe. Component-by-component construction of good lattice rules with a composite number of points. J. Complexity, 18: 943946, 2002.

[Kuo03] F.Y. Kuo. Component-by-component constructions achieve the optimal rate of convergence for multivariate integration in weighted korobov and sobolev spaces. J. Complexity, 19: 301-320, 2003.

[Lar87] G. Larcher. A best lower bound for good lattice points. Monatsh. Math., 104: 45-51, 1987.

[NC05] D. Nuyens and R. Cools. Fast component-by-component construction of rank-1 lattice rules with a non-prime number of points. J. Complexity, 22: 4-28, 2005.

[Nie78] H. Niederreiter. Existence of good lattice points in the sense of Hlawka. Monatsh. Math., 86: 203-219, 1978.

[Nie92] H. Niederreiter. Random number generation and quasi-Monte Carlo methods. SIAM, Philadelphia, 1992.

[SJ94] I.H. Sloan and S. Joe. Lattice methods for multiple integration. Clarendon Press, Oxford, 1994.

[SJ07] V. Sinescu and S. Joe. Good lattice rules based on the general weighted star discrepancy. Math. Comp., 76: 989-1004, 2007.

[SW98] I.H. Sloan and H. Woźniakowski. When are quasi-monte carlo algorithms efficient for high dimensional integrals? J. Complexity, 14: 1-33, 1998.

[Zar68] S.K. Zaremba. Some applications of multidimensional integration by parts. Ann. Polon. Math., 21: 85-96, 1968. 